\title{
Solid Pseudo-papillary Neoplasm of the Pancreas:A Rare Cause of Acute Pancreatitis
}

\section{Michael A. Lopez ${ }^{1^{*}}$, Michael Sand ${ }^{1}$ and Matthew D'Alesssio ${ }^{2}$}

${ }^{1}$ Nova Southeastern University College of Osteopathic Medicine, Davie, FL, USA

${ }^{2}$ Bethesda Memorial Hospital, Boynton Beach, FL, USA

\begin{abstract}
Solid pseudo-papillary neoplasms of the pancreas are rare tumors that typically affect women in the second to fourth decade of life. Although they are known to carry a low malignant potential and carry a favorable prognosis once removed, the presentation and treatment options can differ depending on the location and structures involved. In this case a 23 year-old female presented with an unusual cause for acute pancreatitis.

A high index of suspicion and through diagnostic studies revealed a pseudo-papillary neoplasm in the tail of the pancreas obstructing the pancreatic duct. Definitive treatment included laparoscopic resection of the tail of the pancreas and spleen.
\end{abstract}

Keywords:Pseudo-papillary neoplasm; Frantz tumor; Acute pancreatitis; Pancreas; Surgical oncology

\section{Introduction}

Solid pseudo-papillary neoplasm, also known as a Frantz's tumor, is a rare tumor that typically affects women in the second to fourth decades of life [1]. It is characterized by a well-encapsulated mass that causes a wide variety of local symptoms. These neoplasms have a low malignant potential and usually carry a favorable prognosis [2]. The most common presentation is usually vague, nonspecific abdominal pain. Other symptoms may include nausea, vomiting, and early satiety due to mass effect from the tumor compressing local structures $[2,3]$. Although these tumors typically follow an indolent course, approximately $15 \%$ of cases are malignant with subsequent metastases, almost all of which involve the liver. It is important to identify these tumors early because timely surgery is associated with a $95 \%$ survival rate. We present a rare case of a solid pseudo-papillary neoplasm in a 23 year-old patient presenting with abdominal pain, which was successfully treated with surgical resection.

\section{Case Presentation}

A 23- year-old Haitian-American female, in good overall health, presented to the emergency department of a community hospital with severe abdominal pain. The pain began the night prior to presentation and had not subsided since its onset. The pain was localized to the midepigastric region, and radiated to the left lower quadrant. She described the pain as severe and throbbing, and described a constant feeling of nausea, but denied any vomiting during the episode. She reported no fever, chills, chest pain or shortness of breath. She denied ever suffering from these symptoms in the past.

In the ED, a complete blood count and serum electrolytes were all within normal limits. The serum lipase was elevated at 647 . At this time, she was diagnosed with acute pancreatitis and admitted to the surgical floor, made NPO and started on IV fluids and narcotics.

An ultrasound of the right upper quadrant was obtained to determine if gallstones were cause a possible cause of her pancreatitis. Imaging revealed no gallstones but did identify an unusual mass in the tail of the pancreas. The patient also had an MRCP performed, which confirmed the pancreatic mass causing mild distal pancreatic ductal dilatation (Figure 1).

A CT scan with oral and IV contrast confirmed the presence of a $3 \mathrm{~cm}$, well-circumscribed, low-density mass in the tail of the pancreas, compressing and therefore leading to obstruction of the pancreatic duct (Figure 2). There was noticeable dilatation of the main pancreatic duct distally. This may explain her presentation with acute pancreatitis. The patient then underwent an endoscopic ultrasound and fine needle aspiration which revealed a solid-pseudopapillary neoplasm (Figure 3).

The patient underwent a laparoscopic distal pancreatectomy and splenectomy ten days after her initial presentation (Figure 4). The decision on the timing of surgery was based on the patient's overall clinical picture. By this point the patient had no further abdominal pain, and time was given to allow the acute inflammation around her pancreas to subside. Ultimately, the pancreas was divided with a linear

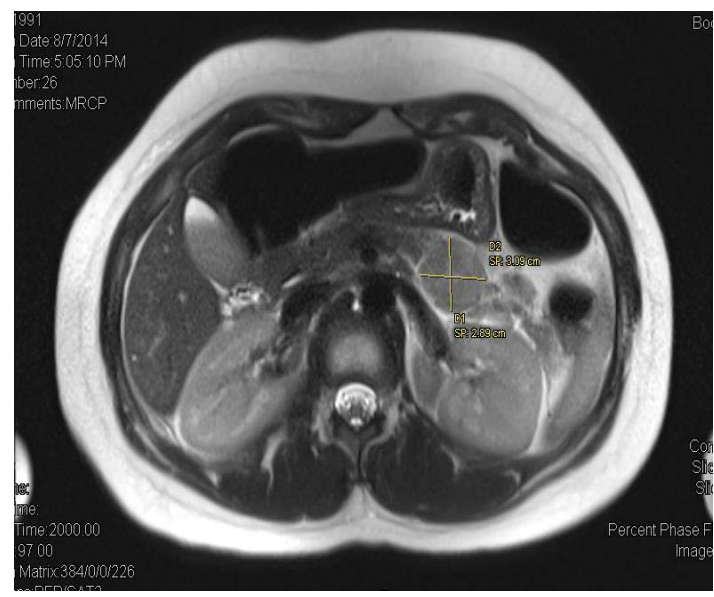

Figure 1: MRI of abdomen demonstrating a mass in the tail of the pancreas.

${ }^{*}$ Corresponding author: Lopez MA, Nova Southeastern University College of Osteopathic Medicine, Davie, FL, USA, Tel: 561-777-2441; E-mail: Mikelopez1004@ gmail.com

Received July 21, 2015; Accepted August 11, 2015; Published August 18, 2015

Citation: Lopez MA, Sand M, Alesssio MD (2015) Solid Pseudo-papillary Neoplasm of the Pancreas:A Rare Cause of Acute Pancreatitis. Surgery Curr Res 5: 244 doi:10.4172/2161-1076.1000244

Copyright: $\odot 2015$ Lopez MA, et al. This is an open-access article distributed under the terms of the Creative Commons Attribution License, which permits unrestricted use, distribution, and reproduction in any medium, provided the original author and source are credited. 


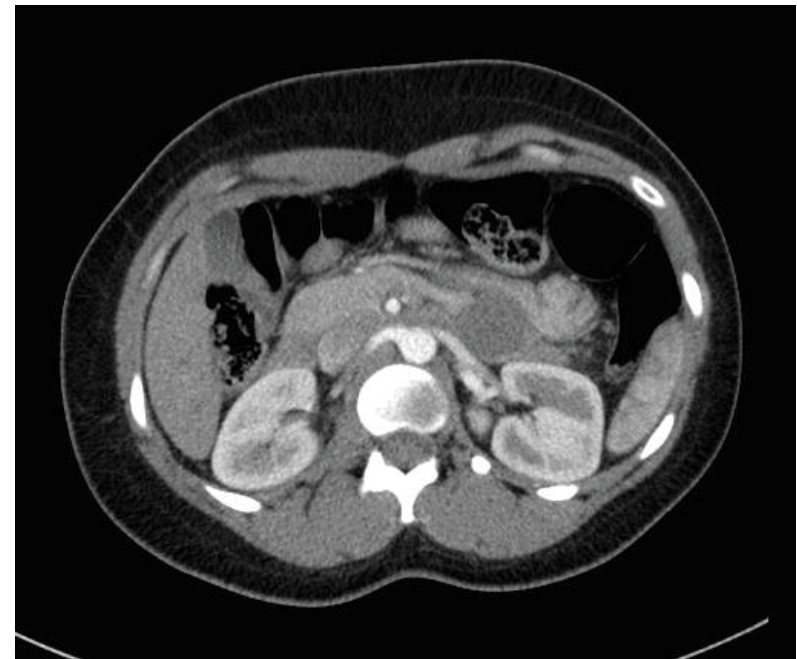

Figure 2: CT scan of abdomen confirming a well-circumscribed $3 \mathrm{~cm}$ mass in the tail of the pancreas.

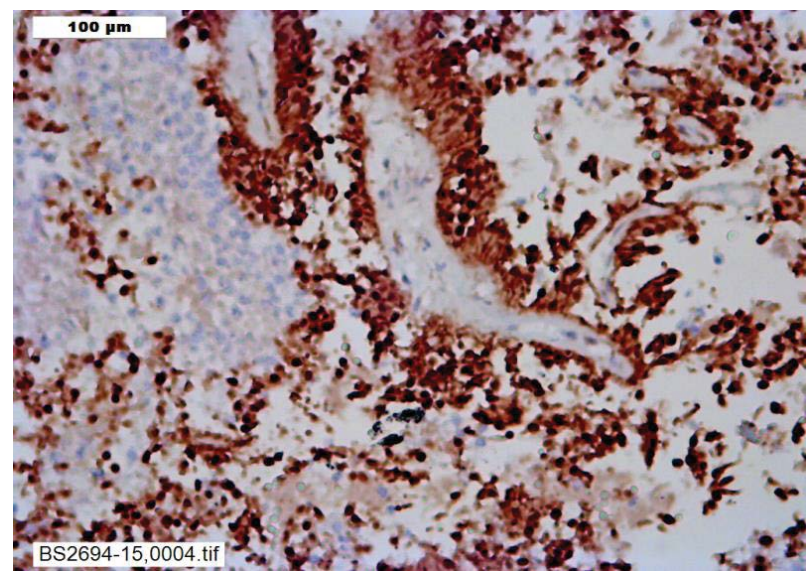

Figure 3: Endoscopic ultrasound-guided fine needle aspiration of a solid pseudopapillary neoplasm of pancreas showing tumor cells lining branching vessels (Diff-Quick stain 20x objective).

cutting stapler with a Gore Seamguard ${ }^{\circledast}$ staple line reinforcement. The patient tolerated the procedure well. Final pathology confirmed a 3.0 $\mathrm{cm}$ solid-pseudopapillary tumor without dysplastic features. There were negative margins at the pancreatic resection margin, and no involved lymph nodes. The patient was discharged five days later with no complications.

\section{Discussion}

Solid pseudo-papillary neoplasms of the pancreas are rare, lowgrade malignancies, that make up less that $1 \%$ of pancreatic tumors, and often affect women in their second to fourth decades of life. It was first described by Frantz in 1959 as a "papillary tumor, benign or malignant" and in 1993, the World Health Organization defined it as "solid pseudopapillary tumors" of the exocrine pancreas [4]. Grossly, these are large, sharply marginated and well-encapsulated pancreatic masses that demonstrate variable degrees of hemorrhage, necrosis and cystic degeneration. On light microscopy, the tumor cells are

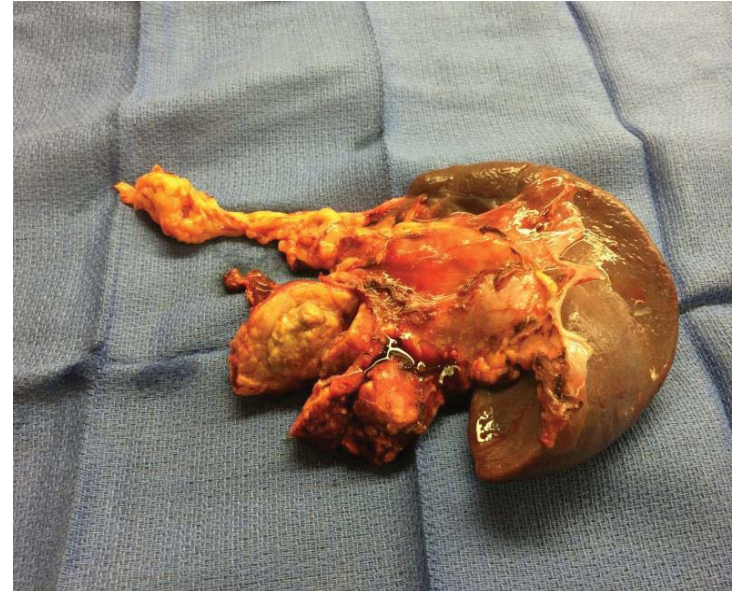

Figure 4: Gross specimen of distal pancreas and spleen removed laparoscopically.

arranged in solid sheets, microcysts and pseudopapillae. The solid sheets contain uniform, polygonal cells within a vascular network. Pseudopapillae are formed by a central thin walled blood vessel surrounded by several layers of dropped neoplastic cells [5]. Histologic findings often include eosinophilic globules, foam cells and cholesterol crystals. Immunohistochemistry shows a distinct pattern that does not correlate to either exocrine or endocrine pancreatic cell types. Solid pseudopapillary neoplasms are positive for CD56, CD10, alpha 1-antitrypsin, neuron specific enolase and vimetinin [5].

Computed tomography is the imaging modality of choice for evaluation of the pancreatic mass and the surrounding structures, which allows for determining if it can be surgically resected [6]. These lesions can be difficult to differentiate due to its heterogeneous composition of cystic and mucinous properties. Because of the tumors varying composition, endoscopic ultrasound and biopsy is the ideal method for a definitive diagnosis. In a series by Sushanth et al, 36 patients with solid pseudopapillary neoplasms had pre-operative CT scans. $39 \%$ had tumor localized to the head of the pancreas, $19 \%$ to the body and $39 \%$ to the tail. Only $3 \%$ have multifocal disease of the head and tail [1].

The definitive treatment for solid pseudopapillary neoplasms is surgical resection in order to both relieve symptoms and eliminated any potential metastatic spread. Because long-term survival can be achieved with radical surgical intervention, aggressive surgical therapy is encouraged once a diagnosis is made [7]. A pancreaticoduodenectomy is required for those lesions within the head of the pancreas, and distal pancreatectomy with or without splenectomy is the treatment of choice for tumors of the pancreatic body and tail [8].

The role of chemotherapy for metastatic solid pseudopapillary neoplasms is unclear. Its use has only been described in selected cases of involving unrespectable metastatic lesions. Gemcitabine based regimens have been used with some success in these rare situations [8].

\section{References}

1. Afridi SA, Lazaryan AM, Marangos IP, Rosok BI, Fretland AA, et al. (2014) Laparoscopic Surgery for Solid Pseudopapillary Tumor of the Pancreas. JSLS 18: $236-242$.

2. Vollmer CM Jr, Dixon E, Grant DR. (2003) Management of a solid pseudopapillary tumor of the pancreas with liver metastases. HPB (Oxford) 5: 264-267. 
Citation: Lopez MA, Sand M, Alesssio MD (2015) Solid Pseudo-papillary Neoplasm of the Pancreas:A Rare Cause of Acute Pancreatitis. Surgery Curr Res 5: 244. doi:10.4172/2161-1076.1000244

3. Patil TB, Shrikhande SV, Kanhere HA, Saoji RR, Ramadwar MR, et al. (2006) Solid pseudopapillary neoplasm of the pancreas: a single institution experience of 14 cases. HPB 8: 148-150.

4. De Castro SM, Singhal D, Aronson DC, Busch OR, van Gulik TM, et al. (2007) Management of solid-pseudopapillary neoplasms of the pancreas: a comparison with standard pancreatic neoplasms. World J Surg 31: 1130-1135.

5. Vilaca A, Rodrigues P, Scigliano H, Pinto J, Reis A. (2011) Solid Pseudopapillary Tumor of the Pancreas: a rare and probably misdiagnosed neoplasm. J Radiol Case Rep 5: 24-34.

6. Choi JY, Kim MJ, Kim JH, Kim SH, Lim JS, et al. (2006) Solid pseudopapillary tumor of the pancreas: typical and atypical manifestations. AJR Am J Roentgenol 187: 178-186.

7. Chang H, Gong Y, Xu J, Su Z, Qin C, et al. (2010) Clinical Strategy for the Management of Solid Pseudopapillary Tumor of the Pancreas: Aggressive or Less? Int J Med Sci 7: 309-313.

8. Reddy S, Cameron JL, Scudiere J, Hruban RH, Fishman EK, et al. (2009) Surgical management of solid-pseudopapillary neoplasms of the pancreas (Franz or Hamoudi tumors): a large single-institutional series. J Am Coll Surg 208: 950-957. 